\title{
Analysis of Reliability of Electric Automation Equipment
}

\author{
Kai Xu \\ Liaoning Jianzhu Vocational College, Liaoyang, Liaoning, 111099
}

Keywords: electric automation equipment; reliability and durability; economy status

\begin{abstract}
With the continuous improvement of China's overall strength and the continuous improvement of the country's economic level, the social economy is also constantly and rapidly growing. At the same time, the reliability of automation control equipment in our country has become more and more complex and changeable due to the continuous development of electrical automation. The country needs to continuously improve the reliability of electrical automation control equipment, and also regulate the reliability test of the electronic control power distribution department. Relevant personnel must continuously strengthen the reliability of electrical automation control equipment and make continuous efforts to increase the level of electrical automation. This paper focuses on the significance of the improvement of electrical automation control equipment and the reliability of electrical automation control equipment to improve the reliability of electrical automation control equipment.
\end{abstract}

\section{Introduction}

In recent years, the development trend of electrical engineering has gradually become more automated. Regardless of the manufacturer or the user, the reliability of electrical automation control equipment is extremely important. Reliability refers to the accurate and reasonable completion of tasks in a specific environment and time. The relevant staff mainly analyzes the reliability problems of electrical automation control equipment, through scientific and effective analysis, find out reasonable control measures, and then continuously improve the reliability of electrical automation control equipment, so that enterprises in the competition is so fierce The emergence of the market ultimately improves the economic benefits and social benefits of construction companies.

\section{The Significance of Reliability Analysis of Electrical Automation Equipment}

Understanding and mastering the significance of doing a good job in the reliability analysis of electrified equipment can not only effectively improve the enthusiasm and enthusiasm of the relevant staff, but also the content that the relevant staff must master, and on the current situation, do a good job of electrical automation equipment The significance of reliability analysis is mainly reflected in the following three aspects: (1) It contributes to the improvement of national comprehensive national strength. The reason why the electrical automation equipment reliability analysis work is conducive to the promotion of the country's overall national strength is because the power companies occupy a very important position in the development of China's national economy; Secondly, the development of electrical automation equipment reliability technology is also To a certain extent, it represents the level of science and technology of the entire country. At the same time, the reliability technology of electrical automation equipment is an important research topic for the relevant staff of power companies. (2) It contributes to the improvement of product quality. Reliability is the core factor in the product quality assurance of electrical automation control equipment. At the same time, the performance and reliability of a product reflect the quality of the product. In this process, reliability plays a major role in it, that is, It is said that only if the reliability of the product is effectively improved, the user can buy the rest assured that the use of peace of mind, but also can effectively reduce the maintenance costs of the product, for the improvement of product credibility and the business economic benefits are all very effective. (3) It will help improve 
the competitiveness of the company. In today's era, the market competition of electrical automation equipment is very fierce, and in order to effectively improve the competitiveness of the market, more customers need to improve the quality and performance of the product, which means that the customer needs a highly reliable equipment product. At the same time, this is also the key and decisive factor in the competition of enterprises. Therefore, the analysis of the reliability of electrical automation equipment will help enterprises to effectively improve their market competitiveness.

\section{Problems in Reliability Analysis of Electrical Automation Control Equipment}

As far as the current situation is concerned, although more and more companies are gradually realizing the important role of the reliability analysis of electrical automation control equipment, there are still some problems and deficiencies in the actual implementation process. (1) The device components are uneven. With the continuous development of China's electrical technology, there are more and more manufacturers of electrical equipment in the market. In the face of fierce market competition, each manufacturer gains more economic benefits and society in order to improve its competitiveness. Benefits began to over-pursue the quantity and type of products, and neglected the quality and reliability of products. Many products with poor reliability and poor quality were mixed into the market. In addition, the related market manufacturers have a mixed bag, the product performance is single, the phenomenon of surplus products is more obvious, the manufacturers will focus more on how to reduce the cost of the product, which in turn makes the reliability of the product difficult to guarantee. (2) Maintenance of electrical automation control equipment needs to be improved. Due to the operation of electrical automation equipment, relatively speaking, there is a certain degree of complexity, so the need for professional knowledge, professional skills and relatively high staff to complete. However, the actual situation is that there are some new and young professional staff with insufficient professional skills in the maintenance of electrical automation control equipment. Because the professional knowledge and work experience of these staff are not enough, due to human factors in the actual operation process will be There are such problems, if the equipment is not effectively maintained for a long time, it will greatly reduce its service life.

Unqualified quality of equipment components In a broad market environment, there are numerous manufacturers. When each manufacturer produces electrical components in electrical automation control equipment, quality requirements and standards vary. Especially those manufacturers with small production scale do not have a sound quality management system and cannot supervise and restrict the production process. As a result, there are defects and loopholes in the production quality of components. In addition, coupled with the impact of the socialist market economic system, there is a vicious competition among manufacturers. In order to reduce costs and expenses, regardless of the quality of production, due to the unqualified quality of equipment components, it directly affects the reliability of electrical automation control equipment.

Because some companies cannot regularly perform maintenance and inspection work, the reliability of electrical automation control equipment cannot be guaranteed. Once there is a defect in the maintenance and inspection work, the electrical automation control equipment will be exposed to the phenomenon of overload operation, which will not only damage the equipment quality, but also shorten the service life of the equipment. In addition, in the process of transportation of electrical automation control equipment, due to lack of necessary protective measures, the equipment is subject to mechanical effects such as vibration and centrifugal force, and then the phenomenon of damage occurs, which poses a serious threat to the reliability of the equipment. . Human factors mainly have the following two points: 1 The operation is not standardized. Newly arrived technicians do not have enough professional knowledge, not enough operating experience, and often do not operate equipment in accordance with the specifications. Some operators have professional knowledge and experience, but they also have various mistakes and mistakes, thus affecting the equipment. Use; 2 lack of regular maintenance.

The electrical automation control equipment needs a specific environment during the operation process. In this process, it will inevitably be affected by external environmental factors. Under 
normal circumstances, the external environment factors mainly refer to the weather factors. If the electrical automation control equipment operates in a high-humidity environment, malfunctions such as insensitive sensing will occur; if the electrical automation control equipment operates in high temperature weather, the equipment components It will be prone to fever and affect its normal operation. This shows that in order to ensure the normal operation of electrical automation control equipment, we must control the operating environment.

\section{Effective Measures to Strengthen the Reliability of Electrical Automation Control Equipment}

The reliability of electrical automation control equipment will be affected by the level of equipment design, so we need to increase design efforts. First of all, when recruiting, you need to hire a professional design staff with a higher level of professionalism to fully understand the production environment and carry out design work. Secondly, design according to the characteristics of the equipment to ensure that the design program is scientific and reasonable. Only by fully understanding the equipment materials, components, functions, and performance requirements can the reliability of electrical automation control equipment be guaranteed. When selecting electronic components, it is necessary to increase the selection of standard components to ensure their accuracy and tightness. Before selecting the electronic components, it is necessary to make reasonable selections of the specifications and models of the manufacturer and the electronic components, and it is not possible to select electronic components that do not meet the standard in order to reduce the cost and expenses. At the same time, we must also do a good job in the detection of electronic components, the test data will be accurately recorded in order to query in the future.

Before the use of electrical automation control equipment, it must be strictly tested on its safety performance, but also to increase the daily maintenance of the equipment to avoid the phenomenon of overloaded equipment. Do regular inspections, and if problems are found, take appropriate measures to resolve them and avoid serious problems. Once equipment is not effectively inspected, reliability is greatly reduced. Therefore, no matter how hard the working environment, equipment maintenance work must not be sloppy, should be strictly in accordance with the standard operation, the replacement of some aging parts in time, so as not to bring more problems, thereby extending the life of the equipment. For some equipments with large workloads, regular inspections are made and corresponding inspection reports are made. This can analyze the causes as soon as possible when the equipment fails, and do not affect the operation as much as possible.

In the process of reliability operation of electrical automation equipment, human factors can not be ignored. In this regard, it is necessary to pay attention to the following aspects: First, staff members must undergo rigorous training before taking up posts. They have a comprehensive and in-depth understanding of the electrical automation control equipment, and accurately grasp the structure of the equipment as well as various functions; When assessing the performance of large staff, it is necessary to focus on the work of the operation. Once irregularities are found, they need to be promptly proposed and corrected. Only when the technical level meets the actual standards, can the job be performed, avoid mistakes in personnel operations, and enhance the reliability of electrical automation control equipment.

Electric automation control equipment is easily affected by the operating environment. For high-humidity environments, it affects the components. If it is in a low-temperature and humid environment, excessive moisture will cause short-circuit faults and damage to components. Therefore, corresponding protective measures must be taken, such as isolating the equipment from the environment. For high-temperature environments, the method of cooling can be adopted to prevent the equipment from being exposed to the high-temperature operating environment for a long period of time, and to perform the heat dissipation maintenance of the equipment. Because the temperature has a direct influence on the stability and reliability of the electronic equipment, the electrical automation equipment with large power will generate thermal energy during operation. Once the external temperature is too high, the internal temperature will be difficult to spread out. In this case, labor is needed for the equipment. Heat dissipation. By adopting reasonable protective 
measures, the reliability of the equipment can be greatly improved, which in turn enables the equipment to operate normally, thereby prolonging its service life.

\section{Conclusion}

The analysis and research on the reliability of electrical automation equipment plays an important role in promoting the development of power companies and the development of the entire national economy. Doing a good job in reliability analysis of electrical automation equipment has become a direction and goal that the relevant staff has been working hard for, but as far as the current situation is concerned, the problems still existing in the reliability analysis process of electrical automation equipment need to be improved and optimized, so the relevant staff We must be clear about our own responsibilities and responsibilities. We must be good at finding problems, analyzing problems, and resolving problems in practical work, so as to effectively solve the reliability problems of electrified equipment.

\section{References}

[1] Wang Xiuting, Guan Shunfeng, Hu Shuhua. Construction of China's auto industry technology innovation platform [J]. WuJournal of Han University of Technology (Information and Management Engineering), 2002, (6).

[2] Gu Xiaoan, Li Binbin, Gu Baoyan. Research on the Influence of Technological Innovation on Economic Development-ComeAn Empirical Analysis of Korean Automobile Industry[J]. Shanghai Economic Research,2007, (12).

[3] Li Xiaodong, Zhao Jian, Hou Wenbin, et al. Multi-level open experimental teaching body for vehicle engineering Department of Construction [J]. Experimental Technology and Management, 2011, (10).

[4] Zhai Zhigang. Application of UAV Remote Sensing Technology in Surveying and Mapping Engineering[J]. Residential with real estate, 2016, (18): 256.

[5] Zhang Weilin, Song Xiuchen. Discussion on reliability testing method of electrical automation control equipment [J]. SM Industry Management and Technology, 2009, 7. 